\title{
ON THE CLASS OF SQUARE PETRIE MATRICES INDUCED BY CYCLIC PERMUTATIONS
}

\author{
BAU-SEN DU
}

Received 2 September 2003

\begin{abstract}
Let $n \geq 2$ be an integer and let $P=\{1,2, \ldots, n, n+1\}$. Let $Z_{p}$ denote the finite field $\{0,1,2, \ldots$, $p-1$ \}, where $p \geq 2$ is a prime. Then every map $\sigma$ on $P$ determines a real $n \times n$ Petrie matrix $A_{\sigma}$ which is known to contain information on the dynamical properties such as topological entropy and the Artin-Mazur zeta function of the linearization of $\sigma$. In this paper, we show that if $\sigma$ is a cyclic permutation on $P$, then all such matrices $A_{\sigma}$ are similar to one another over $Z_{2}$ (but not over $Z_{p}$ for any prime $p \geq 3$ ) and their characteristic polynomials over $Z_{2}$ are all equal to $\sum_{k=0}^{n} x^{k}$. As a consequence, we obtain that if $\sigma$ is a cyclic permutation on $P$, then the coefficients of the characteristic polynomial of $A_{\sigma}$ are all odd integers and hence nonzero.
\end{abstract}

2000 Mathematics Subject Classification: 15A33, 15A36.

1. Introduction. Throughout this paper, let $n \geq 2$ be a fixed integer and let $P=$ $\{1,2, \ldots, n, n+1\}$. For every integer $1 \leq i \leq n$, let $J_{i}=[i, i+1]$. Let $\sigma$ be a map from $P$ into itself. The linearization of $\sigma$ on $P$ is defined as the continuous map $f_{\sigma}$ from $[1, n+1]$ into itself such that $f_{\sigma}(k)=\sigma(k)$ for every integer $1 \leq k \leq n+1$ and $f_{\sigma}$ is linear on $J_{i}$ for every integer $1 \leq i \leq n$. Let $A_{\sigma}=\left(a_{i j}\right)$ be the real $n \times n$ matrix defined by $a_{i j}=1$ if $f_{\sigma}\left(J_{i}\right) \supset J_{j}$ and $a_{i j}=0$ otherwise. The definition of $A_{\sigma}$ may seem opaque. But if we take $J_{i}$ 's as the vertices of a directed graph and draw an arrow from the vertex $J_{i}$ to the vertex $J_{j}$ if $f_{\sigma}\left(J_{i}\right) \supset J_{j}$, then $A_{\sigma}$ will be the adjacency matrix [4, page 17] of the resulting directed graph. For example, the adjacency matrix of the cyclic permutation $\sigma: 1 \rightarrow 2 \rightarrow 5 \rightarrow 4 \rightarrow 3 \rightarrow 1$ is given as

$$
A_{\sigma}=\left[\begin{array}{llll}
0 & 1 & 1 & 1 \\
1 & 1 & 1 & 1 \\
1 & 1 & 0 & 0 \\
0 & 0 & 1 & 0
\end{array}\right] .
$$

In the theory of discrete dynamical systems on the interval, this adjacency matrix $A_{\sigma}$ turns out to contain much information on the dynamical properties of the map $f_{\sigma}$. For example, for some special types (including cyclic permutations) of $\sigma$, if $x^{n}+\sum_{k=0}^{n-1} a_{k} x^{k}$ is the characteristic polynomial of $A_{\sigma}$, then it is shown in [6] that the Artin-Mazur zeta function $\zeta(z)$ [2] of $f_{\sigma}$ is $\zeta(z)=1 /\left(1+\sum_{k=1}^{n} a_{n-k} z^{k}\right)$. On the other hand, it follows from [1, Theorem 4.4.5, page 222] or [4, Proposition 19, page 204] that the topological entropy of $f_{\sigma}$ equals $\max \{0, \log \lambda\}$, where $\lambda$ is the maximal eigenvalue of $A_{\sigma}$. Since every cyclic graph defines a communication channel, as defined by Shannon, we can claim that the logarithm of the largest eigenvalue of $A_{\sigma}$ gives its channel capacity. This motivates further investigation of such matrices $A_{\sigma}$. 
Due to the continuity of $f_{\sigma}$, it is clear that such matrices $A_{\sigma}$ have entries either zeros or ones such that the ones in each row occur consecutively. Actually, we have $a_{i j}=1$ for all $a_{i} \leq j \leq b_{i}-1$, where $a_{i}=\min \left\{f_{\sigma}(i), f_{\sigma}(i+1)\right\}$ and $b_{i}=\max \left\{f_{\sigma}(i), f_{\sigma}(i+1)\right\}$, and $a_{i j}=0$ elsewhere. For our purpose, we define a Petrie matrix [5] to be a matrix whose entries are either zeros or ones such that the ones in each row occur consecutively. So, the matrix $A_{\sigma}$ induced by a map $\sigma$ on $P$ is a square Petrie matrix whose determinant is easily seen (by induction) [7] to be either 0 or \pm 1 . For any prime number $p \geq 2$, let $Z_{p}=\{0,1,2, \ldots, p-1\}$ denote the usual finite field and let $W_{Z_{p}}=\left\{\sum_{i=1}^{n} r_{i} J_{i} \mid r_{i} \in Z_{p}, 1 \leq\right.$ $i \leq n\}$ be the $n$-dimensional vector space over $Z_{p}$ with $\left\{J_{i} \mid 1 \leq i \leq n\right\}$ as a set of basis. Then the matrix $A_{\sigma}(\bmod 2)$ defines a linear transformation $\psi_{\sigma}$ on $W_{Z_{2}}$ such that, for every integer $1 \leq i \leq n, \psi_{\sigma}\left(J_{i}\right)=\sum_{j=1}^{n} a_{i j} J_{j}$.

If both $\sigma$ and $\rho$ are just permutations on $P$, then it is easy to see that $A_{\sigma}$ may not be similar to $A_{\rho}$ over $Z_{2}$. But if both $\sigma$ and $\rho$ are cyclic permutations on $P$, then we show, in this paper, that $A_{\sigma}$ is similar to $A_{\rho}$ over $Z_{2}$ (but $A_{\sigma}$ may not be similar to $A_{\rho}$ over $Z_{p}$ for any prime $p \geq 3$ ) and their characteristic polynomials over $Z_{2}$ are all equal to $\sum_{k=0}^{n} x^{k}$. As a consequence, we obtain that if $\sigma$ is a cyclic permutation, then the coefficients of the characteristic polynomial of $A_{\sigma}$ are all odd integers and hence nonzero (not true in general if $\sigma$ is not cyclic) with constant term \pm 1 .

2. On the Petrie matrix $A_{\sigma}$ over $Z_{2}$ with any map $\sigma$ on $P$. In the following, we let $[x: y]$ denote the closed interval on the real line with $x$ and $y$ as endpoints. For integers $1 \leq k<j \leq n+1$, we let $[k, j]$ denote the element $\sum_{i=k}^{j-1} J_{i}$ of $W_{Z_{2}}$ and call $k$ and $j$ the endpoints (this terminology will be used in the proof of Theorem 3.2 in Section 3) of the element $\sum_{i=k}^{j-1} J_{i}$. Part (2) of the following lemma is proved in [4, pages 22-23], which will be needed in Section 3. Here, we present a different proof (see also [3]).

Lemma 2.1. Let $n, P, J_{i}$ 's, $\sigma, f_{\sigma}, W_{Z_{2}}, \psi_{\sigma}, A_{\sigma}$ be defined as in Section 1. Let $\rho$ be a map from $P$ into itself and let $\psi_{\rho}$ and $A_{\rho}$ be defined similarly. Then the following hold.

(1) Let $1 \leq k<j \leq n+1$ be any integers. Then for any element $[k, j]=\sum_{i=k}^{j-1} J_{i}$ in $W_{Z_{2}}$, $\psi_{\sigma}([k, j])=\left[f_{\sigma}(k): f_{\sigma}(j)\right]$.

(2) $\psi_{\rho} \circ \psi_{\sigma}=\psi_{\rho \circ \sigma}$ and $\left(A_{\sigma}\right)\left(A_{\rho}\right) \equiv A_{\rho \circ \sigma}(\bmod 2)$. Consequently, if $\sigma$ is a permutation on $P$, then $\psi_{\sigma}$ is invertible with inverse $\psi_{\sigma^{-1}}$ and $A_{\sigma}$ is nonsingular with determinant \pm 1 .

Proof. It follows from the definition of $\psi_{\sigma}$ in Section 1 that $\psi_{\sigma}\left(J_{i}\right)=\left[f_{\sigma}(i)\right.$ : $\left.f_{\sigma}(i+1)\right]$ for every integer $1 \leq i \leq n$. Thus, we obtain that $\psi_{\sigma}([k, j])=\psi_{\sigma}\left(\sum_{i=k}^{j-1} J_{i}\right)=$ $\sum_{i=k}^{j-1} \psi_{\sigma}\left(J_{i}\right)=\sum_{i=k}^{j-1}\left[f_{\sigma}(i): f_{\sigma}(i+1)\right]=\left[f_{\sigma}(k): f_{\sigma}(j)\right]$ since $1+1=0$ in $Z_{2}$. This proves part (1).

By part $(1), \psi_{\sigma}([k, j])=\left[f_{\sigma}(k): f_{\sigma}(j)\right]$. Similarly, $\psi_{\rho}([k, j])=\left[f_{\rho}(k): f_{\rho}(j)\right]$. So, $\left(\psi_{\rho} \circ \psi_{\sigma}\right)\left(J_{i}\right)=\psi_{\rho}\left(\left[f_{\sigma}(i): f_{\sigma}(i+1)\right]\right)=\left[f_{\rho}\left(f_{\sigma}(i)\right): f_{\rho}\left(f_{\sigma}(i+1)\right)\right]=[(\rho \circ \sigma)(i):$ $(\rho \circ \sigma)(i+1)]=\psi_{\rho \circ \sigma}\left(J_{i}\right)$ since, on the finite set $P, f_{\sigma}=\sigma$ and $f_{\rho}=\rho$. This shows that $\psi_{\rho} \circ \psi_{\sigma}=\psi_{\rho \circ \sigma}$ on $W_{Z_{2}}$. Thus, if $\sigma$ is a permutation on $P$, then $\psi_{\sigma^{-1}} \circ \psi_{\sigma}=\psi_{\sigma^{-1} \circ \sigma}$ is the identity map on $W_{Z_{2}}$, and so $\psi_{\sigma}$ is an invertible linear transformation on $W_{Z_{2}}$ with inverse $\psi_{\sigma^{-1}}$. The rest of part (2) can be easily proved and is omitted. This proves part (2) and completes the proof of Lemma 2.1. 
3. On the Petrie matrix $A_{\sigma}$ with any cyclic permutation $\sigma$ on $P$. We will need the following elementary result. We include its proof for completeness.

LEMMA 3.1. Let $1 \leq j \leq n$ be any fixed integer and let $b$ denote the greatest common divisor of $j$ and $n+1$. Let $s=(n+1) / b$. For every integer $1 \leq k \leq s-1$, let $1 \leq m_{k} \leq n$ be the unique integer such that $k j \equiv m_{k}(\bmod n+1)$. Then the $m_{k}$ 's are all distinct and $\left\{m_{k} \mid 1 \leq k \leq s-1\right\}=\{k b \mid 1 \leq k \leq s-1\}$.

Proof. Let $B=\left\{m_{k} \mid 1 \leq k \leq s-1\right\}$ and $C=\{k b \mid 1 \leq k \leq s-1\}$. For every integer $1 \leq k \leq s-1$, since $j / b$ and $(n+1) / b$ are relatively prime, the congruence equation $(j / b) x \equiv k(\bmod (n+1) / b)$ has a solution in $1 \leq x \leq s-1=(n+1) / b-1$. Consequently, for every integer $1 \leq k \leq s-1$, the congruence equation $j x \equiv k b(\bmod n+1)$ has a solution in $1 \leq x \leq s-1$. Since $1 \leq k b \leq n$ for every $1 \leq k \leq s-1$, we obtain that $C \subset B$. Since both $B$ and $C$ contain exactly $s-1$ elements, we have $B=C$. That is, $\left\{m_{k} \mid 1 \leq k \leq s-1\right\}=\{k b \mid 1 \leq k \leq s-1\}$. This completes the proof.

Theorem 3.2. Let $n, P, J_{i}$ 's, $\sigma, f_{\sigma}, W_{Z_{2}}, \psi_{\sigma}, A_{\sigma}$ be defined as in Section 1. Assume that $\sigma$ is also a cyclic permutation on $P$. Then the following hold.

(1) For every integer $1 \leq i \leq n, \sum_{k=0}^{n} \psi_{\sigma}^{k}\left(J_{i}\right)=\mathbf{0}$. Consequently, $\sum_{k=0}^{n} \psi_{\sigma}^{k}(w)=\mathbf{0}$ for all $w \in W_{Z_{2}}$.

(2) Let $1 \leq i \leq n-1$ and $1 \leq j_{j} \leq n$ be two fixed integers such that $1 \leq i<f_{\sigma}^{j}(i) \leq n$ and let $J=\left[i, f_{\sigma}^{j}(i)\right]=\sum_{k=i}^{f_{\sigma}^{j}(i)-1} J_{k}$. Assume that $j$ and $n+1$ are relatively prime. Then the set $\left\{\psi_{\sigma}^{k}(J) \mid 0 \leq k \leq n-1\right\}$ is a basis for $W_{Z_{2}}$.

(3) For any cyclic permutations $\sigma$ and $\rho$ on $P, \psi_{\sigma}$ and $\psi_{\rho}$ are similar on $W_{Z_{2}}$. Consequently, the Petrie matrices over $Z_{2}$ of all cyclic permutations on $P$ are similar to one another and have the same characteristic polynomial $\sum_{k=0}^{n} x^{k}$.

(4) The coefficients of the characteristic polynomial of $A_{\sigma}$ are all odd integers (and hence nonzero) with constant term \pm 1 .

REMARK 3.3. Part (3) of the above theorem does not hold if the Petrie matrices of cyclic permutations are over the finite field $Z_{p}$ for any prime $p \geq 3$. For example, if $P=\{1,2,3,4,5\}, \sigma$ denotes the cyclic permutation $1 \rightarrow 2 \rightarrow 5 \rightarrow 4 \rightarrow 3 \rightarrow 1$, and $\rho$ denotes the cyclic permutation $1 \rightarrow 2 \rightarrow 3 \rightarrow 4 \rightarrow 5 \rightarrow 1$, then $A_{\sigma}$ and $A_{\rho}$ are not similar over $Z_{p}$ for any prime $p \geq 3$ because the characteristic polynomials of $A_{\sigma}$ and $A_{\rho}$ are $x^{4}-x^{3}-3 x^{2}-3 x-1$ and $x^{4}-x^{3}-x^{2}-x-1$, respectively, which are distinct over $Z_{p}$ for any prime $p \geq 3$.

Proof. For any fixed integer $1 \leq i \leq n$, let $1 \leq j \leq n$ be the unique integer such that $f_{\sigma}^{j}(i)=i+1$, and so $J_{i}=[i, i+1]=\left[i, f_{\sigma}^{j}(i)\right]$. Let $b$ denote the greatest common divisor of $j$ and $n+1$ and let $s=(n+1) / b$. For every integer $1 \leq k \leq s-1$, let $1 \leq m_{k} \leq n$ be the unique integer such that $k j \equiv m_{k}(\bmod n+1)$. Then, by Lemma 3.1, we obtain that $\left\{m_{k} \mid 1 \leq k \leq s-1\right\}=\{k b \mid 1 \leq k \leq s-1\}$. Let $m_{0}=0$. Then $\left\{m_{k} \mid 0 \leq k \leq s-1\right\}=\{k b \mid$ $0 \leq k \leq s-1\}$. Hence, the set $\{0,1,2,3, \ldots, n-1, n\}$ is the disjoint union of the sets $\left\{m_{k}+\right.$ $m \mid 0 \leq k \leq s-1\}, 0 \leq m \leq b-1$. Therefore, $\sum_{k=0}^{s-1} \psi_{\sigma}^{m_{k}}\left(J_{i}\right)=\sum_{k=0}^{s-1} \psi_{\sigma}^{k j}\left(J_{i}\right)$ (since $k j \equiv$ $\left.m_{k}(\bmod n+1)\right)=\left[i: f_{\sigma}^{j}(i)\right]+\left[f_{\sigma}^{j}(i): f_{\sigma}^{2 j}(i)\right]+\left[f_{\sigma}^{2 j}(i): f_{\sigma}^{3 j}(i)\right]+\cdots+\left[f_{\sigma}^{(s-2) j}(i):\right.$ $\left.f_{\sigma}^{(s-1) j}(i)\right]+\left[f_{\sigma}^{(s-1) j}(i): i\right]=\mathbf{0}$. So, $\sum_{\ell=0}^{n} \psi_{\sigma}^{\ell}\left(J_{i}\right)=\sum_{m=0}^{b-1} \psi_{\sigma}^{m}\left(\sum_{k=0}^{s-1} \psi_{\sigma}^{m_{k}}\left(J_{i}\right)\right)=\mathbf{0}$. This proves part (1). 
For the proof of part (2), we first show that if $E$ is a nonempty subset of $\{1,2,3, \ldots$, $n-1, n\}$ such that $J+\sum_{k \in E} \psi_{\sigma}^{k}(J)=\mathbf{0}$, then $E=\{1,2,3, \ldots, n-1, n\}$. Indeed, for every integer $1 \leq k \leq n$, let $1 \leq m_{k} \leq n$ be the unique integer such that $k j \equiv m_{k}(\bmod n+1)$. Assume that $m_{1}=j \notin E$. Then, for any $m \in E, m \neq 0, j$. Since $\psi_{\sigma}^{m}(J)=\psi_{\sigma}^{m}\left(\left[i, f_{\sigma}^{j}(i)\right]\right)=$ $\left[f_{\sigma}^{m}(i): f_{\sigma}^{m+j}(i)\right]$, the endpoints of $\psi_{\sigma}^{m}(J)$ do not contain the point $f_{\sigma}^{j}(i)$. Thus, in the expression of $\psi_{\sigma}^{m}(J)$ as a sum of the basis elements $J_{k}$ 's, it contains either both the basis elements $J_{f_{\sigma}^{j}(i)-1}$ and $J_{f_{\sigma}^{j}(i)}$ or none of them. But, since $J=\left[i, f_{\sigma}^{j}(i)\right]=J_{i}+J_{i+1}+$ $\cdots+J_{f_{\sigma}^{j}(i)-1}$ contains the element $J_{f_{\sigma}^{j}(i)-1}$, but not the element $J_{f_{\sigma}^{j}(i)}$, in its expression as a sum of the basis elements $J_{k}$ 's, we obtain that in the expression of $J+\sum_{m \in E} \psi_{\sigma}^{m}(J)$ as a sum of the basis elements $J_{k}$ 's, the coefficient of $J_{f_{\sigma}^{j}(i)-1}$ is different from that of $J_{f_{\sigma}^{j}(i)}$ by 1 . This implies that $J+\sum_{m \in E} \psi_{\sigma}^{m}(J) \neq \mathbf{0}$, which is a contradiction. Therefore, $m_{1}=j \in E$.

Thus,

$$
\begin{aligned}
\mathbf{0} & =J+\sum_{m \in E} \psi_{\sigma}^{m}(J) \\
& =J+\psi_{\sigma}^{j}(J)+\sum_{m \in E \backslash\left\{m_{1}\right\}} \psi_{\sigma}^{m}(J) \\
& =\left[i, f_{\sigma}^{j}(i)\right]+\left[f_{\sigma}^{j}(i): f_{\sigma}^{2 j}(i)\right]+\sum_{m \in E \backslash\left\{m_{1}\right\}} \psi_{\sigma}^{m}(J) \\
& =\left[i: f_{\sigma}^{2 j}(i)\right]+\sum_{m \in E \backslash\left\{m_{1}\right\}} \psi_{\sigma}^{m}(J) .
\end{aligned}
$$

Proceeding in this manner finitely many times, we obtain that $\left\{m_{1}, m_{2}, \ldots, m_{n-1}\right\} \subset E$ and

$$
\begin{aligned}
\mathbf{0}= & J+\sum_{m \in E} \psi_{\sigma}^{m}(J) \\
= & {\left[i: f_{\sigma}^{2 j}(i)\right]+\sum_{m \in E \backslash\left\{m_{1}\right\}} \psi_{\sigma}^{m}(J) } \\
= & {\left[i: f_{\sigma}^{3 j}(i)\right]+\sum_{m \in E \backslash\left\{m_{1}, m_{2}\right\}} \psi_{\sigma}^{m}(J) } \\
= & \cdots=\left[i: f_{\sigma}^{n j}(i)\right] \\
& +\sum_{m \in E \backslash\left\{m_{1}, m_{2}, \ldots, m_{n-1}\right\}} \psi_{\sigma}^{m}(J) .
\end{aligned}
$$

In particular, $\mathbf{0}=\left[i: f_{\sigma}^{n j}(i)\right]+\sum_{m \in E \backslash\left\{m_{1}, m_{2}, \ldots, m_{n-1}\right\}} \psi_{\sigma}^{m}(J)$. If $m \in E$ and $m \neq m_{n}$, then, as above, since $m \neq 0$ and $m \neq m_{n} \equiv n j(\bmod n+1)$, the endpoints of $\psi_{\sigma}^{m}(J)$ do not contain the point $f_{\sigma}^{n j}(i)$. Hence, in the expression of $\psi_{\sigma}^{m}(J)$ as a sum of the basis elements $J_{k}$ 's, it contains either both the basis elements $J_{f_{\sigma}^{n j}(i)-1}$ and $J_{f_{\sigma}^{n j}(i)}$ or none of them. But, since $\left[i, f_{\sigma}^{n j}(i)\right]=J_{i}+J_{i+1}+\cdots+J_{f_{\sigma}^{n j}(i)-1}$ contains the element $J_{f_{\sigma}^{n j}(i)-1}$, not the element $J_{f_{\sigma}^{n j}(i)}$, in its expression as a sum of the basis elements $J_{k}$ 's, we obtain that in the expression of $\left[i: f_{\sigma}^{n j}(i)\right]+\sum_{m \in E \backslash\left\{m_{1}, m_{2}, \ldots, m_{n-1}\right\}} \psi_{\sigma}^{m}(J)$ as a sum of the basis elements $J_{k}$ 's, the coefficient of $J_{f_{\sigma}^{n j}(i)-1}$ is different from that 
of $J_{f_{\sigma}^{n j}(i)}$ by 1 . This implies that $\left[i: f_{\sigma}^{n j}(i)\right]+\sum_{m \in E \backslash\left\{m_{1}, m_{2}, \ldots, m_{n-1}\right\}} \psi_{\sigma}^{m}(J) \neq \mathbf{0}$, which is a contradiction. Thus, $m_{n}=n j \in E$. Since, by assumption, $j$ and $n+1$ are relatively prime, we see that, by Lemma 3.1, $\left\{m_{1}, m_{2}, \ldots, m_{n}\right\}=\{1,2, \ldots, n-1, n\}$. Since $\left\{m_{1}, m_{2}, \ldots, m_{n}\right\} \subset E \subset\{1,2, \ldots, n-1, n\}$, we obtain that $E=\{1,2, \ldots, n-1, n\}$. This proves our assertion.

Now, assume that $\sum_{k=0}^{n-1} \alpha(k) \psi_{\sigma}^{k}(J)=\mathbf{0}$, where $\alpha(k)=0$ or 1 in $Z_{2}, 0 \leq k \leq n-1$. If $\alpha(0)=0$ and $\alpha(\ell) \neq 0$ for some integer $1 \leq \ell<n-1$, let $\ell$ be the smallest such integer; then, since $\psi_{\sigma}$ is invertible by Lemma 2.1(2), we obtain that $J+\sum_{k=1}^{n-\ell-1} \alpha(k) \psi_{\sigma}^{k}(J)=$ 0. So, without loss of generality, we may assume that $\alpha(0) \neq 0$. That is, we assume that $J+\sum_{k=1}^{n-1} \alpha(k) \psi_{\sigma}^{k}(J)=\mathbf{0}$. Let $E=\{k \mid 1 \leq k \leq n-1, \alpha(k) \neq 0\}$. Then, we have $J+\sum_{k \in E} \psi_{\sigma}^{k}(J)=\mathbf{0}$. But then it follows from what we have just proved above that $E=\{1,2, \ldots, n-1, n\}$. This contradicts the assumption that $E \subset\{1,2, \ldots, n-1\}$. So, the set $\left\{\psi_{\sigma}^{k}(J) \mid 0 \leq k \leq n-1\right\}$ is linearly independent and hence, by [8], is a basis for $W_{Z_{2}}$. This proves part (2).

Let $\theta$ denote the cyclic permutation $1 \rightarrow 2 \rightarrow 3 \rightarrow \cdots \rightarrow i \rightarrow i+1 \rightarrow \cdots \rightarrow n \rightarrow n+1 \rightarrow 1$ on $P$ and let $\sigma$ be any cyclic permutation on $P$. Choose any fixed integer $1 \leq j \leq n$ such that $j$ and $n+1$ are relatively prime and let $J=\left[1, f_{\sigma}^{j}(1)\right]$. Then, by part (2), the set $\left\{\psi_{\sigma}^{k}(J) \mid 0 \leq k \leq n-1\right\}$ is a basis for $W_{Z_{2}}$. Let $\phi$ be the linear transformation on $W_{Z_{2}}$ defined by $\phi\left(J_{k}\right)=\psi_{\sigma}^{k-1}(J), 1 \leq k \leq n$. Then $\phi$ is an isomorphism on $W_{Z_{2}}$. Furthermore, $\left(\phi \circ \psi_{\theta}\right)\left(J_{n}\right)=\phi\left(\sum_{k=1}^{n} J_{k}\right)=\sum_{k=1}^{n} \phi\left(J_{k}\right)=\sum_{k=1}^{n} \psi_{\sigma}^{k-1}(J)=\psi_{\sigma}^{n}(J)$ (by part (1)) = $\psi_{\sigma}\left(\psi_{\sigma}^{n-1}(J)\right)=\psi_{\sigma}\left(\phi\left(J_{n}\right)\right)=\left(\psi_{\sigma} \circ \phi\right)\left(J_{n}\right)$ and, for every integer $1 \leq k \leq n-1,(\phi \circ$ $\left.\psi_{\theta}\right)\left(J_{k}\right)=\phi\left(\psi_{\theta}\left(J_{k}\right)\right)=\phi\left(J_{k+1}\right)=\psi_{\sigma}^{k}(J)=\psi_{\sigma}\left(\psi_{\sigma}^{k-1}(J)\right)=\psi_{\sigma}\left(\phi\left(J_{k}\right)\right)=\left(\psi_{\sigma} \circ \phi\right)\left(J_{k}\right)$. Thus, $\psi_{\sigma}$ is similar to $\psi_{\theta}$ through $\phi$. Since the property of similarity is obviously transitive, we obtain that if $\rho$ is any cyclic permutation on $P$, then $\psi_{\sigma}$ and $\psi_{\rho}$ are similar on $W_{Z_{2}}$. Consequently, by [8], the Petrie matrices (over $Z_{2}$ ) of all cyclic permutations on $P$ are similar to one another and so have the same characteristic polynomial $\sum_{k=0}^{n} x^{k}$ since $\sum_{k=0}^{n} x^{k}$ is easily verified to be the characteristic polynomial of the Petrie matrix $A_{\theta}$ over $Z_{2}$. This proves part (3).

Finally, let $\sigma$ be a cyclic permutation on $P$. Since $A_{\sigma}$ is a real $n \times n$ matrix with entries either zeros or ones, the coefficients of the characteristic polynomial of $A_{\sigma}$ are all integers. By taking every entry in $A_{\sigma}$ modulo 2 and applying part (3) and the fact that the determinants of Petrie matrices are either 0 or \pm 1 , we obtain that the characteristic polynomial of $A_{\sigma}(\bmod 2)$ is equal to $\sum_{k=0}^{n} x^{k}$. Consequently, the coefficients of the characteristic polynomial of $A_{\sigma}$ are all odd integers with constant term \pm 1 . This proves part (4) and completes the proof of Theorem 3.2.

ACKNOwLEDGMent. The author would like to thank Professor Peter Jau-Shyong Shiue for his interest and many helpful suggestions which led to the improvement of this paper.

\section{REFERENCES}

[1] L. Alsedà, J. Llibre, and M. Misiurewicz, Combinatorial Dynamics and Entropy in Dimension One, 2nd ed., Advanced Series in Nonlinear Dynamics, vol. 5, World Scientific Publishing, New Jersey, 2000.

[2] M. Artin and B. Mazur, On periodic points, Ann. of Math. (2) 81 (1965), 82-99. 
[3] C. Bernhardt, A proof of Sharkovsky's theorem, J. Difference Equ. Appl. 9 (2003), no. 3-4, 373-379.

[4] L. S. Block and W. A. Coppel, Dynamics in One Dimension, Lecture Notes in Mathematics, vol. 1513, Springer-Verlag, Berlin, 1992.

[5] R. A. Brualdi and H. J. Ryser, Combinatorial Matrix Theory, Encyclopedia of Mathematics and Its Applications, vol. 39, Cambridge University Press, Cambridge, 1991.

[6] B.-S. Du, The linearisations of cyclic permutations have rational zeta functions, Bull. Austral. Math. Soc. 62 (2000), no. 2, 287-295.

[7] M. Gordon and E. M. Wilkinson, Determinants of Petrie matrices, Pacific J. Math. 51 (1974), 451-453.

[8] I. N. Herstein, Topics in Algebra, Blaisdell Publishing, New York, 1964.

Bau-Sen Du: Institute of Mathematics, Academia Sinica, Taipei 11529, Taiwan

E-mail address: mabsdu@sinica.edu. tw 


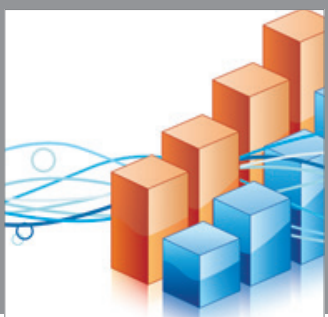

Advances in

Operations Research

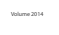

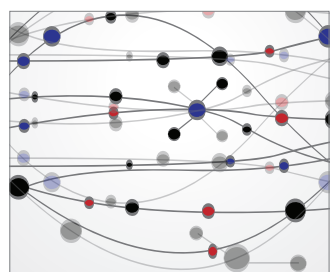

\section{The Scientific} World Journal
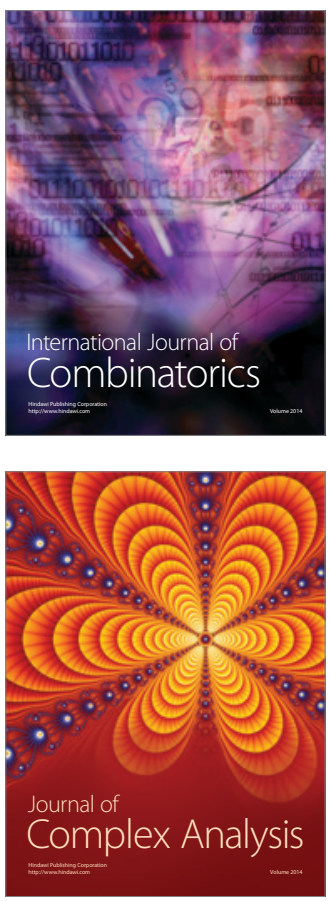

International Journal of

Mathematics and

Mathematical

Sciences
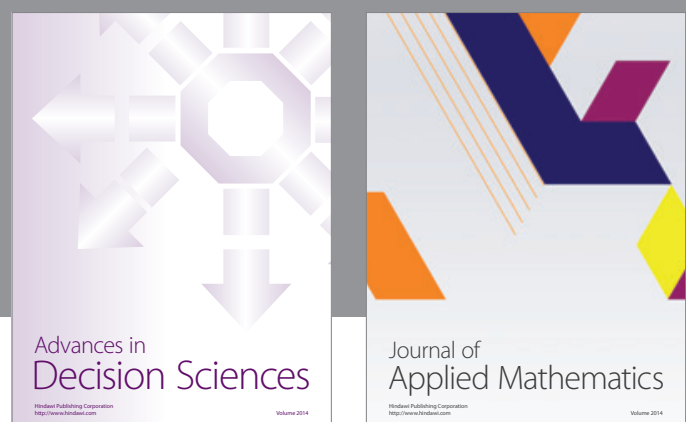

Journal of

Applied Mathematics
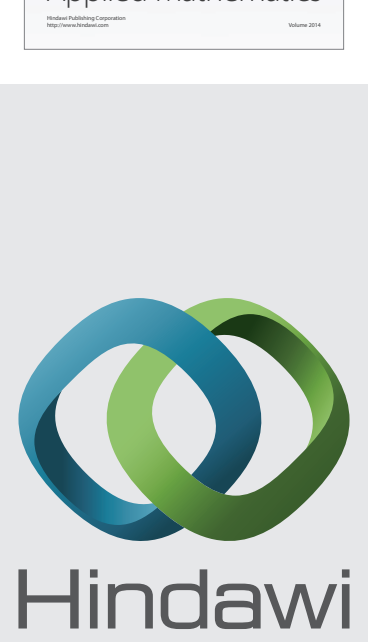

Submit your manuscripts at http://www.hindawi.com
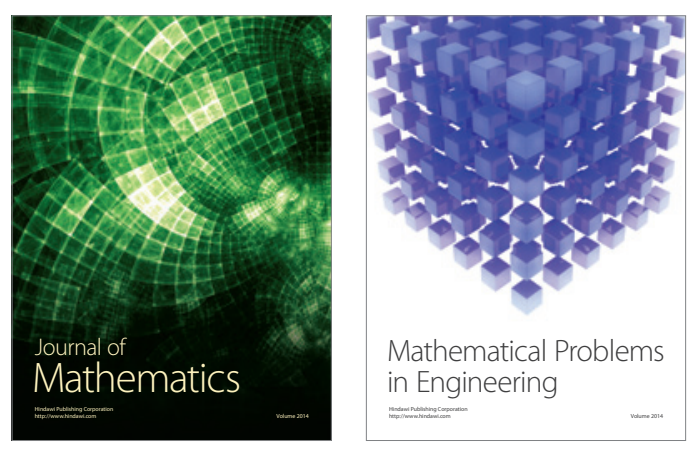

Mathematical Problems in Engineering
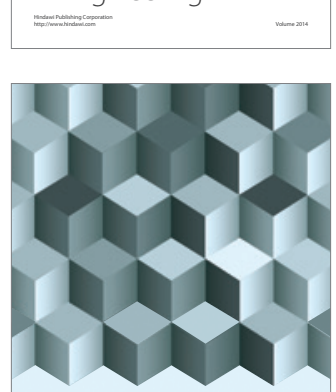

Journal of

Function Spaces
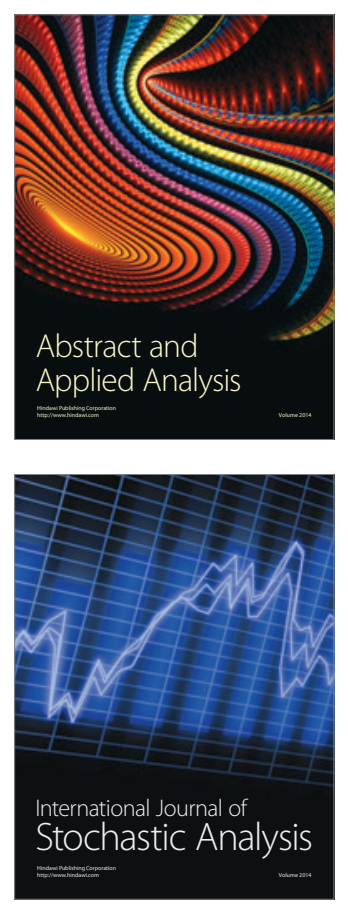

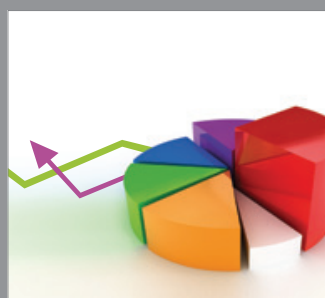

ournal of

Probability and Statistics

Promensencen
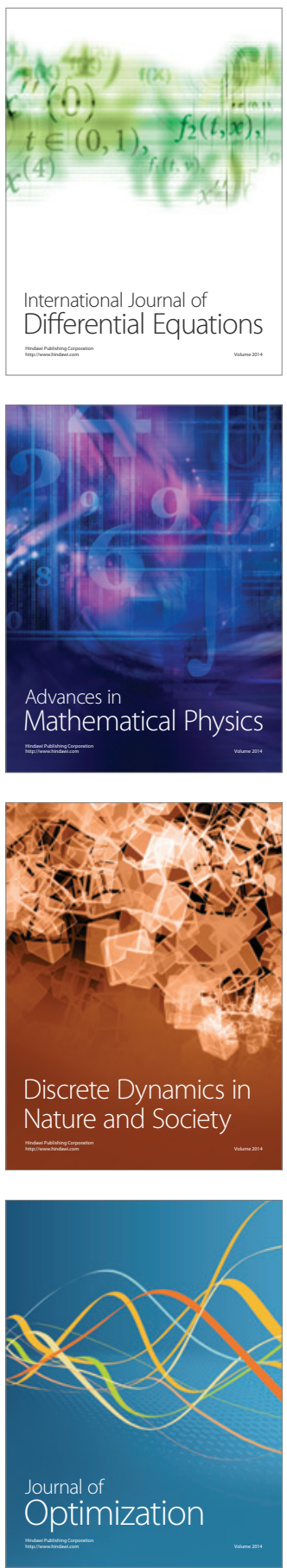